\title{
Pluripotency and Chimera Competence of an Embryonic Stem Cell Line from the Sea Perch (Lateolabrax japonicus)
}

\author{
Song-Lin Chen, ${ }^{1}$ Zhen-Xia Sha, ${ }_{1}^{1}$ Han-Qing Ye, ${ }^{1}$ Yang Liu, ${ }^{1}$ Yong-Sheng Tian, ${ }^{1}$ \\ Yunhan Hong, ${ }^{2}$ Qi-Sheng Tang ${ }^{1}$ \\ ${ }^{1}$ Yellow Sea Fisheries Research Institute, Chinese Academy of Fisheries Sciences, Key Lab for Sustainable Utilization of Marine \\ Fisheries Resources, Ministry of Agriculture, Qingdao 266071, China \\ ${ }^{2}$ Department of Biological Sciences, National University of Singapore, 10 Kent Ridge Crescent, Singapore 119260
}

Received: 8 April 2006 / Accepted: 14 July 2006 / Published online: 30 November 2006

\begin{abstract}
A stable GFP-expressing (GFP ${ }^{+}$LJES1) cell strain was developed from the LJES1 cells obtained from sea perch (Lateolabrax japonicus,) embryos. GFP ${ }^{+}$LJES1 cells were induced in vitro by RA to differentiate into a variety of cell types and also had the ability to form embryoid body-like structures in suspension culture. To determine the differentiation potential of LJES1 cells in vivo, GFP ${ }^{+}$LJES1 cells were transplanted into sea perch and zebrafish embryos at midblastula stage. Twenty out of 478 transplanted sea perch embryos contained GFP-expressing LJES1 cells $24 \mathrm{~h}$ after microinjection. Fifteen chimera embryos developed into fry. In these chimeras, the GFP $^{+}$LJES1 cells contributed to a variety of tissues including the head and trunk. In zebrafish, 221 embryos were microinjected with $\mathrm{GFP}^{+}$LJES1 cells and 22 chimera embryos and fries expressing GFP were obtained. Donor $\mathrm{GFP}^{+}$LJES1 cells contributed to various tissues in head and trunk of zebrafish embryos and hatched fry.
\end{abstract}

Keywords: Cell transplantation - chimera embryonic stem cells - pluripotency - sea perch zebrafish

\section{Introduction}

Embryonic stem (ES) cells are undifferentiated cells derived from early animal embryos retaining pluripotency and capability to differentiate (Evans and Kaufman, 1981; Martin, 1981). ES cell lines have

Correspondence to: Song-Lin Chen; E-mail: chensl@ysfri.ac.cn been established in human (Thomson et al., 1998) and mouse (Thomas and Capecchi, 1987; Thompson et al., 1989) and have been used in a variety of areas including gene expression and cell differentiation (Mummery et al., 1990).

ES cells are also a powerful tool in teleost for developmental genetics and gene transfer. Transgenic fish research requires developing of a novel gene transfer approach for achieving site-directed integration of a transgene into the host genome (Hong et al., 1998a; Melamed et al., 2002; Chen et al., 2002a; Rocha et al., 2004; Wadman et al., 2005). To develop ES cell lines and gene targeting technique in fish, extensive attempts have been made in model fish such as zebrafish (Danio rerio; Collodi et al., 1992; Sun et al., 1995; Fan et al., 2004a) and medaka (Oryzias latipes; Wakamatsu et al., 1994; Hong et al., 1996). In commercial fish species, ES-like cell lines have also been described in the gilthead seabream (Sparus aurata; Bejar et al., 2002), sea perch (L. japonicus; Chen et al., 2003a), red sea bream (Chrysophrys major; Chen et al., 2003b), and turbot (Scophthalmus maximus; Holen and Hamre, 2003). The medaka ES cell lines MES1 (Hong et al., 1998b) and Sa BE-1c from seabream (Bejar et al., 2002) were shown to retain a diploid karyotype and the ability to form viable chimeras. Homologous recombination vectors for medaka $p 53$ gene were constructed (Chen et al., 2001, 2002b). Also, a positive-negative selection procedure for gene targeting in fish cells has been established (Chen et al., 2002a; Fan et al., 2006). Most importantly, the availability of zebrafish germline chimeras from embryo primary cell cultures and short-term ES cell culture (Ma et al., 2001; Fan et al., 2004b) and the feasibility of nuclear transplantation for medaka embryonic cells (Wakamatsu et al., 2001) and zebrafish 
(Lee et al., 2002) point to the possibility of developing the ES cell technology in fish.

Sea perch (L. japonicus) is widely cultivated in China and also a potential valued marine model fish. Pluripotent embryonic cell cultures have been developed and differentiation ability in vitro of the normal cell culture LJES1 was demonstrated (Chen et al., 2003a). However, no data are available on the development of GFP-expressing cell strain, its in vitro differentiation ability, and chimera generation. With the aim of establishing gene targeting technique and ES cell-mediated gene transfer approach in sea perch, we report here on the establishment of a GFP-expressing LJES1 cell strain, its in vitro differentiation, and generation of chimeras by cell transplantation.

\section{Materials and Methods}

Sea Perch Embryonic Stem Cell Line and Culture. Sea perch early embryonic cell culture (LJES1) was established previously in our laboratory (Chen et al., 2003a). Routine culture of the LJES1 cells was performed as described (Chen et al., 2003a). Cells were cultured at $24^{\circ} \mathrm{C}$ in a normal atmosphere incubator and the medium was changed every 2 or 3 days.

Transfection of GFP Gene into LJES1 Cells. Plasmid pCMV-EGFP-PAC was previously constructed (Hong, unpublished) and prepared by using the Maxi-prep kit (Qiagen). To optimize transfection condition, LJES1 cells were transfected at passage 15 to 18 with three reagents-Genejammer (Stratagene), Genejuice (Novagen) and Metafectene (Biontex). In brief, the cells were plated at $1.5 \times$ $10^{5}$ cells/well of 12 -well plates and incubated for 16 to $20 \mathrm{~h}$. Transfection was carried out according to the manufacturer's instructions. At 48 to $72 \mathrm{~h}$ after transfection, GFP expression in the cells was detected by fluorescence microscopy (CK40, Olympus). For evaluating transfection efficiency, cells expressing GFP were counted under a fluorescent microscope.

Development of GFP-Expressing LJES1 Cell Strain. At $48 \mathrm{~h}$ after transfection, cells were trypsinized and subcultured in a ratio of 1:6 and incubated. After complete adhesion, cells were treated with 0.6 to $0.8 \mu \mathrm{g} / \mathrm{ml}$ of puromycin and incubated at $24^{\circ} \mathrm{C}$ with regular medium changes. After 3 to 4 weeks of selection culture, some GFP-expressing LJES1 cells formed single colonies. Cell colonies with stronger GFP fluorescence were picked up and propagated in complete medium containing 0.2 to $0.4 \mu \mathrm{g} / \mathrm{ml}$ of puromycin.

In Vitro Differentiation of GFP-Expressing LJES1 Cells. To examine the effect of GFP gene transfection on the differentiation potential of LJES1, the GFP ${ }^{+}$LJES1 cells were inoculated at low density $\left(10^{4}\right.$ cells/well of 12 -well plates $)$ or high density $\left(5 \times 10^{5}\right.$ cells/well of 12 -well plate $)$ and then cultured for 1 to 4 weeks. For inducing differentiation, 0.1 to $1 \mu \mathrm{M}$ all-trans retinoic acid (RA, Sigma) was added to the cell culture 2 days after inoculation. Control cultures were similarly seeded on gelatin-coated wells without retinoic acid.

Formation of Embryoid Bodies. To induce the formation of embryoid bodies (EBs), LJES1 cells were digested into single cells and cultured in suspension on a dish cover according to Mummery and vanden Eijnden-van Raaij (1993) with some modifications. In brief, GFP-expressing LJES1 cells at a concentration of $10^{6} / \mathrm{ml}$ cells were inoculated as mini drops on the inner surface of the dish cover and cultured in suspension at $24^{\circ} \mathrm{C}$. After 2 to 3 days, the suspended cell cultures formed EBs and were transferred to a tissue culture plate for further culture and observation.

Transplantation of $\mathrm{GFP}^{+}$LJES1 Cells into Sea Perch Blastula. Recipient embryos used for cell transplantation were obtained by artificial fertilization. Blastula-stage embryos were rinsed in embryo medium and placed in a depression made in an agarose-covered 60-mm Petri dish.

For preparation of donor cells, $\mathrm{GFP}^{+} \mathrm{LJES} 1$ cells at passage 18 to 20 were harvested and suspended in transplantation medium. Cell transplantation was carried out by using a microinjector (Cell Tram Oil, Eppendorf) connected to an oil hydraulic MMO202ND micromanipulator (Narishige, Japan) under

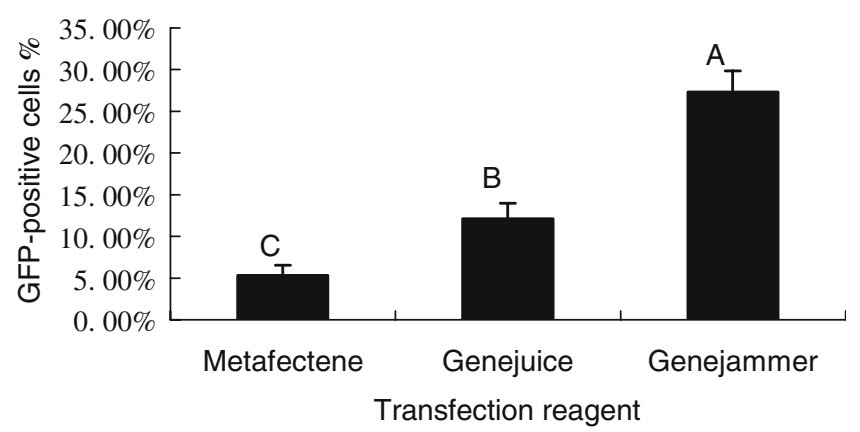

Fig. 1. Comparison of transfection efficiency of various transfection reagents. Values (mean \pm standard error) are from three independent experiments. 

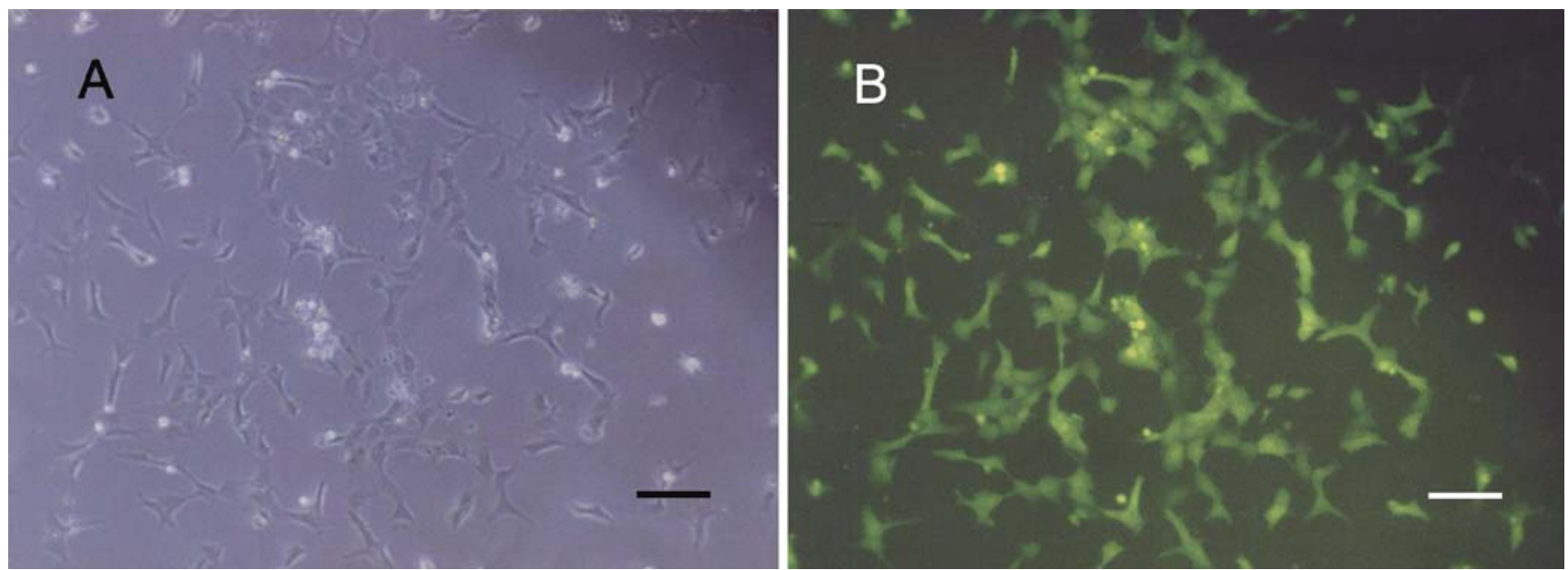

Fig. 2. GFP-expressing LJES1 cells. (A) Bright field (control); (B) dark field. Scale bar $=100 \mu \mathrm{m}$.

a stereoscopic microscope (MZAPO, Leica). Injection needles were made from 1-mm borosilicate glass capillaries with a vertical pipette puller (Narishige, Japan) had a tip with an internal diameter of 15 to $20 \mu \mathrm{m}$. The cell suspension was pipetted onto a flat surface and sucked into the needle. Fifty to one hundred $\mathrm{GFP}^{+} \mathrm{LJES1}$ cells were microinjected into the mid-blastula. The injected embryos and control were incubated in sterilized sea water at $18^{\circ} \mathrm{C}$. To visualize GFP cells, embryos were observed via fluorescence microscopy (CK40, Olympus).

Transplantation of $\mathrm{GFP}^{+} \mathrm{LJES1}$ Cells into Zebrafish Blastula. Wild-type zebrafish were purchased from Nanshan Market (Qingdao, China). They were reared as described in the Zebrafish Book. On the day before transplantation, individual female zebrafish were placed in mating cages with one or two males with a divider separating the female from the males. After initiation of the light cycle, the divider was removed to place the female and the males together. The breeding activity began soon thereafter. Embryos of high quality at the blastula stage were chosen for transplantation.

Cell transplantation was performed as described in the preceding text. The tip of the needles was broken to give an internal diameter of 20 to $50 \mu \mathrm{m}$. Ten to fifty LJES1 cells at passage 28 to 30 were microinjected into recipient embryos between the blastula stage and the $30 \%$-epiboly stage.
Injected embryos were first cultured in Holtfreter's solution $(3.5 \mathrm{~g}$ of $\mathrm{NaCl}, 0.05 \mathrm{~g}$ of $\mathrm{KCl}$, $0.1 \mathrm{~g}$ of $\mathrm{CaCl}_{2}, 0.2 \mathrm{~g}$ of $\mathrm{NaHCO}_{3}$ in $1 \mathrm{~L}$ of sterile water) at $28^{\circ} \mathrm{C}$ for $24 \mathrm{~h}$. Then they were transferred to sterile water and incubated at $28^{\circ} \mathrm{C}$ until hatching. Hatchlings were cultured in tap water.

Statistical Analysis. Data are expressed as means \pm SEM. Transfection efficiencies of the three liposome transfection reagents were analyzed via independent-samples test. A value of $p<0.05$ was considered statistically significant. The statistical analysis was computed using SPSS software.

\section{Results}

Transfection Efficiency of Different Transfection Reagents. The effects of different transfection reagents on transfection efficiency were examined. It was demonstrated that LJES1 cells were successfully transfected with pCMV-EGFP by means of the three liposome transfection reagents-Genejammer, Genejuice, and Metafectene. Among the three reagents, Genejammer resulted in the highest transfection efficiency $(27.3 \%)$ when $0.5 \mu \mathrm{g}$ of the GFP plasmid DNA and $3 \mu \mathrm{l}$ of Genejammer reagent were used (Figure 1). Genejuice generated the efficiency of $12.1 \%$. In contrast, Metafectene resulted in the lowest transfection efficiency $(5.3 \%)$ for the LJES1 cells (Figure 1).

Fig. 3. Differentiation ability in vitro of GFP LJES1. (A) Muscle-like cells under light microscopy; (B) muscle-like cells under fluorescent microscopy; (C) neuron-like cells conglomeration under common light microscopy; (D) neuron-like cell conglomeration under fluorescent microscope; (E) fiber and bipolar cells that were arranged parallel under a common light microscope; (F) fiber-like and bipolar cells that were arranged parallel under a fluorescence microscope; $(\mathbf{G}, \mathbf{H})$ differentiated single neuron-like cells with long axon and arborization under a fluorescence microscope. Magnification: $\times 100$. Scale bars $=$ $100 \mu \mathrm{m}$. 


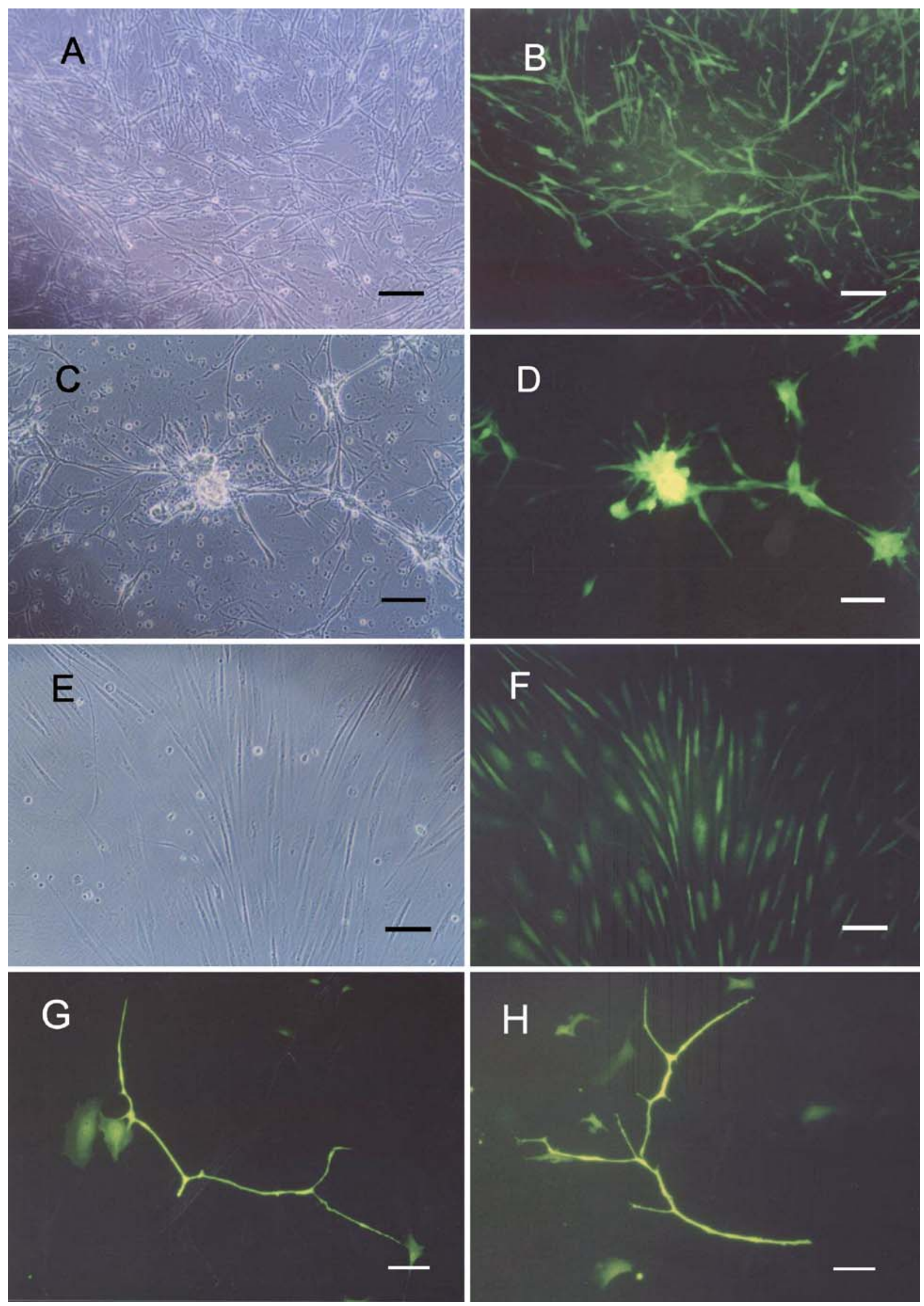



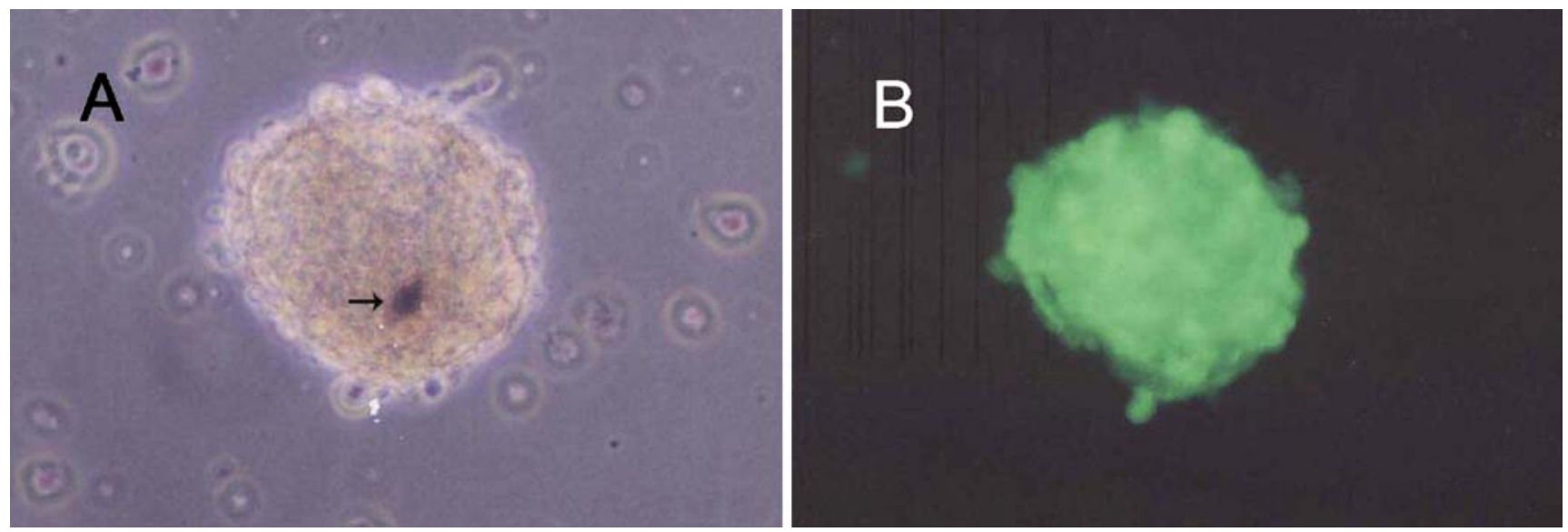

Fig. 4. Formation of embryoid bodies induced from GFP LJES1 cells. (A) Embryoid body with pigment cells (arrowhead) under a common light microscope. (B) Embryoid body under a flourescence microscope. Magnification $\times 100$.

Establishment of GFP-Expressing LJES1 Cell Strain. To develop a GFP-expressing LJES1 cell strain, GFP transfected cells were subcultured in a ratio of 1:6 $48 \mathrm{~h}$ after transfection. Twenty-four hours later, the cells were selected with the drug puromycin. After 3 to 4 weeks of selection, single colonies expressing GFP were formed. One single colony with strong GFP expression was picked up and propagated. After 7 to 10 subcultures, one stable LJES1 cell strain expressing GFP was obtained. All cells from the GFP-positive cell strain express GFP (Figure 2). After culture of more than 60 days with more than 20 passages, GFP ${ }^{+}$LJES1 cells displayed stable growth and ES-like morphology. GFP mRNA transcripts were detected in the GFP ${ }^{+}$LJES1 cells by reverse transcriptase-polymerase chain reaction (RT-PCR; data not shown). Cryopreservation tests demonstrated that $\mathrm{GFP}^{+} \mathrm{LJES} 1$ cells could be preserved in liquid nitrogen $\left(-196^{\circ} \mathrm{C}\right)$ with a survival rate of about $60 \%$.

Differentiation Potential of $\mathrm{GFP}^{+}$LJES1 Cells In Vitro. Differentiation of $\mathrm{GFP}^{+} \mathrm{LJES1}$ cells into a variety of cell types could be induced by all-trans retinoic acid. When $\mathrm{GFP}^{+} \mathrm{LJES} 1$ cells at passage 10 to
30 were treated with retinoic acid, most cells lost their stem cell-like morphology and started differentiation into various cell types, including muscle-like cells, at 7 to 10 days (Figure 3A, B), neuron-like cells at 15 to 20 days (Figure 3C, D) and other unidentified cells. In some cases, 12 to 20 days $\mathrm{GFP}^{+}$LJES1 cells were differentiated into unidentified fiber-like, bipolar cells parallel to each other (Figure 3E, F). Single neuron-like cells with long axons or arborization began to appear 15 to 20 days after induction when cells were inoculated at very low densities (Figure 3G, H). All differentiated cells express GFP.

GFP $^{+}$LJES1 Cells Form EBs. GFP ${ }^{+}$LJES1 cells were capable of forming EBs after 3 days of suspension culture (Figure 4). The EBs showed tight round or ellipse structure. When transferred into a cell culture plate, the cells were able to attach easily to the surface of the cell culture plate. Some pigment cells appeared in some EBs (Figure 4A).

Chemira Production. Sea Perch Chimera from GFP $^{+}$LJES1 Cell Transplantation. The results of chimera production reveal that from 478 micro-

Table 1. Chimera Production from GFP ${ }^{+}$LJES1 Cell Transplantation into Sea Perch

\begin{tabular}{lccccc}
\hline Experiment & $\begin{array}{l}\text { Embryos } \\
\text { injected } n\end{array}$ & $\begin{array}{l}\text { Embryos } \\
\text { survived } n(\%)\end{array}$ & $\begin{array}{l}\text { GFP-expressing } \\
\text { embryos } n(\%)\end{array}$ & $\begin{array}{l}\text { Embryo survival } \\
\text { Hatched chimeras } n(\%)\end{array}$ & $\begin{array}{l}\text { Eontrol (\%) } \\
\text { in con }\end{array}$ \\
\hline 1 & 70 & $31(44.3)$ & $1(3.2)$ & - & 78 \\
2 & 85 & $37(43.5)$ & $1(2.7)$ & $3(3.8)$ & 80 \\
3 & 139 & $78(56.1)$ & $4(5.1)$ & $6(8.7)$ & 74 \\
4 & 103 & $69(67.0)$ & $8(11.6)$ & $5(10.9)$ & 76 \\
5 & 81 & $46(56.8)$ & $6(13.0)$ & $15(5.8)$ & 78 \\
Total & 478 & $261(54.6)$ & $20(7.7)$ & & 76 \\
\hline
\end{tabular}



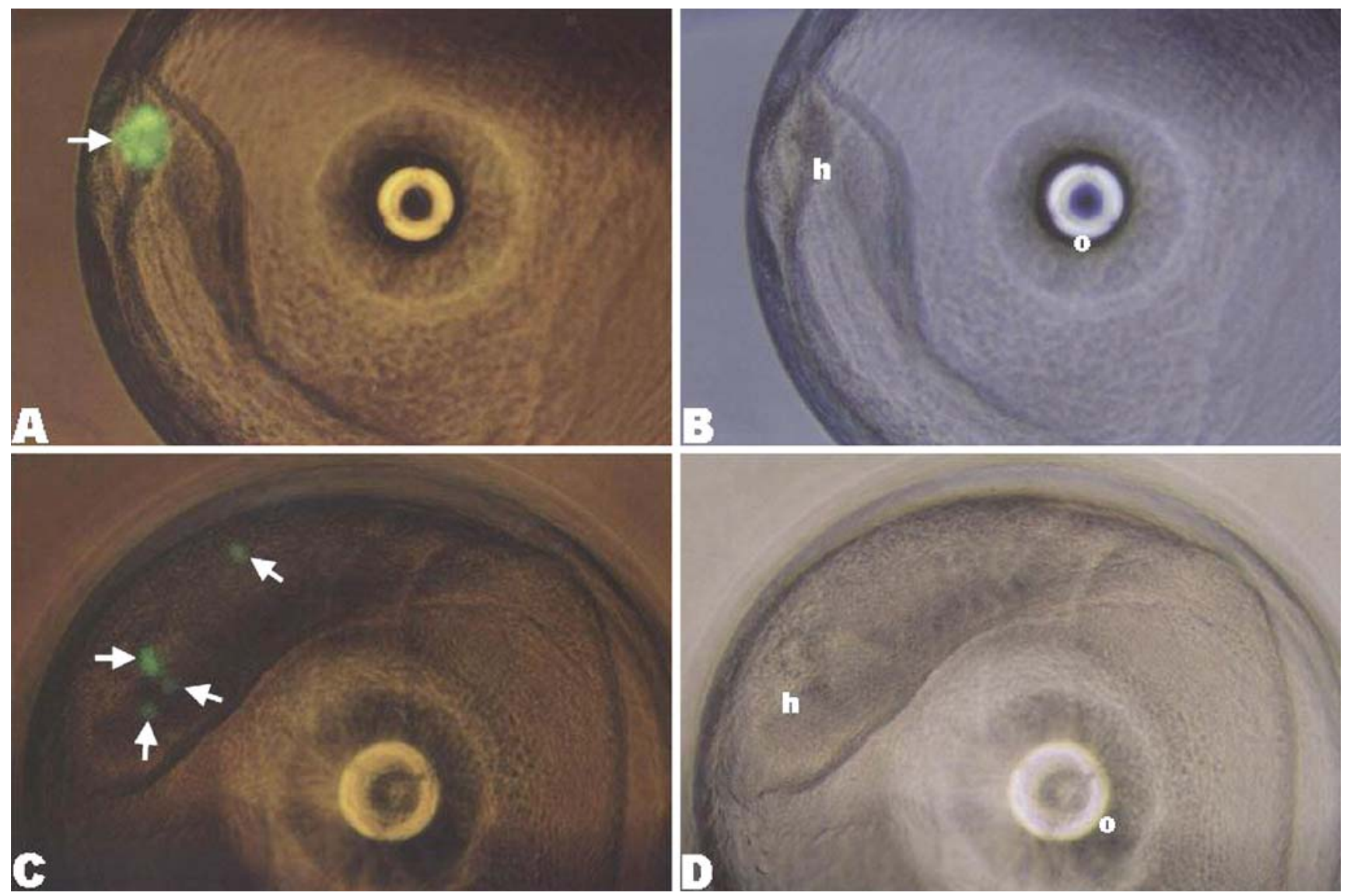

Fig. 5. Chimera embryos of sea perch obtained by transplanting GFP LJES1 cells into sea perch blastula. $(\mathbf{A}, \mathbf{B}) \times 100$, 48-h embryo showing a mass of donor cells in head (arrow). (C, D) 48-h embryo showing donor cells in different compartments (arrow). (A, C) observed under a fluorescence microscope; (B, D) observed under a common microscope. Magnification: $\times 100$; h, head; o, oil ball.

injected embryos 261 embryos (54.6\%) survived at $24 \mathrm{~h}$ (Table 1). From these, 20 expressed GFP at $24 \mathrm{~h}$ after microinjection (Table 1) and 15 of them hatched out. The donor GFP LJES1 cells contributed to various embryonic structures including head and trunk (Figure 5), either concentrated in one (Figure 5A) or in different compartments (Figure 5C).

Zebrafish Chimera from GFP ${ }^{+}$LJES1 Cell Transplantation. Experiments were performed to transplant GFP ${ }^{+}$LJES1 cells into zebrafish embryos. Two hundred and twenty-one zebrafish embryos were microinjected in seven batches of experiments. Survival rate at $24 \mathrm{~h}$ was $61 \%$ (data not shown). Twenty-two chimera embryos and fries expressing GFP were obtained. LJES1 cells contributed widely to embryonic structure in head and trunk (Figure 6). Some GFP $^{+}$LJES1 cells did not integrate well with the host embryo (Figure 6C) in some transplanted embryos. Some GFP ${ }^{+}$LJES1 cells showed good integration with the host embryo and differentiated into muscle-like cells (Figure 6E) or neuron-like cells (Figure 6G) in some chimera embryos. GFP ${ }^{+}$LJES1 cells were found to contribute to muscle tissues in head and tail of some chimera fry at 3 days after hatching (Figure 7).

\section{Discussion}

So far, ES-like cell lines have been established in some model fish (Wakamatsu et al., 1994; Hong et al., 1996; Fan et al., 2004a) and cultured marine fish (Bejar et al., 2002; Chen et al., 2003a,b). However, no report was available on the development and differentiation in vitro of GFP-expressing ES cell strain in cultured fish. Retaining in vitro differentiation ability of ES cells after genetic transfection is essential for developing a gene targeting technique. The present article reports the establishment of a GFP-expressing sea perch ES cell strain $\left(\mathrm{GFP}^{+} \mathrm{LJES} 1\right)$ and demonstrates that the GFP $^{+}$LJES1 cells still exhibited the ability to differentiate in vitro into a variety of cell types such as muscles cells, neuron-like cells, and unidentified cell types, which suggested that the sea perch GFP $^{+}$LJES1 

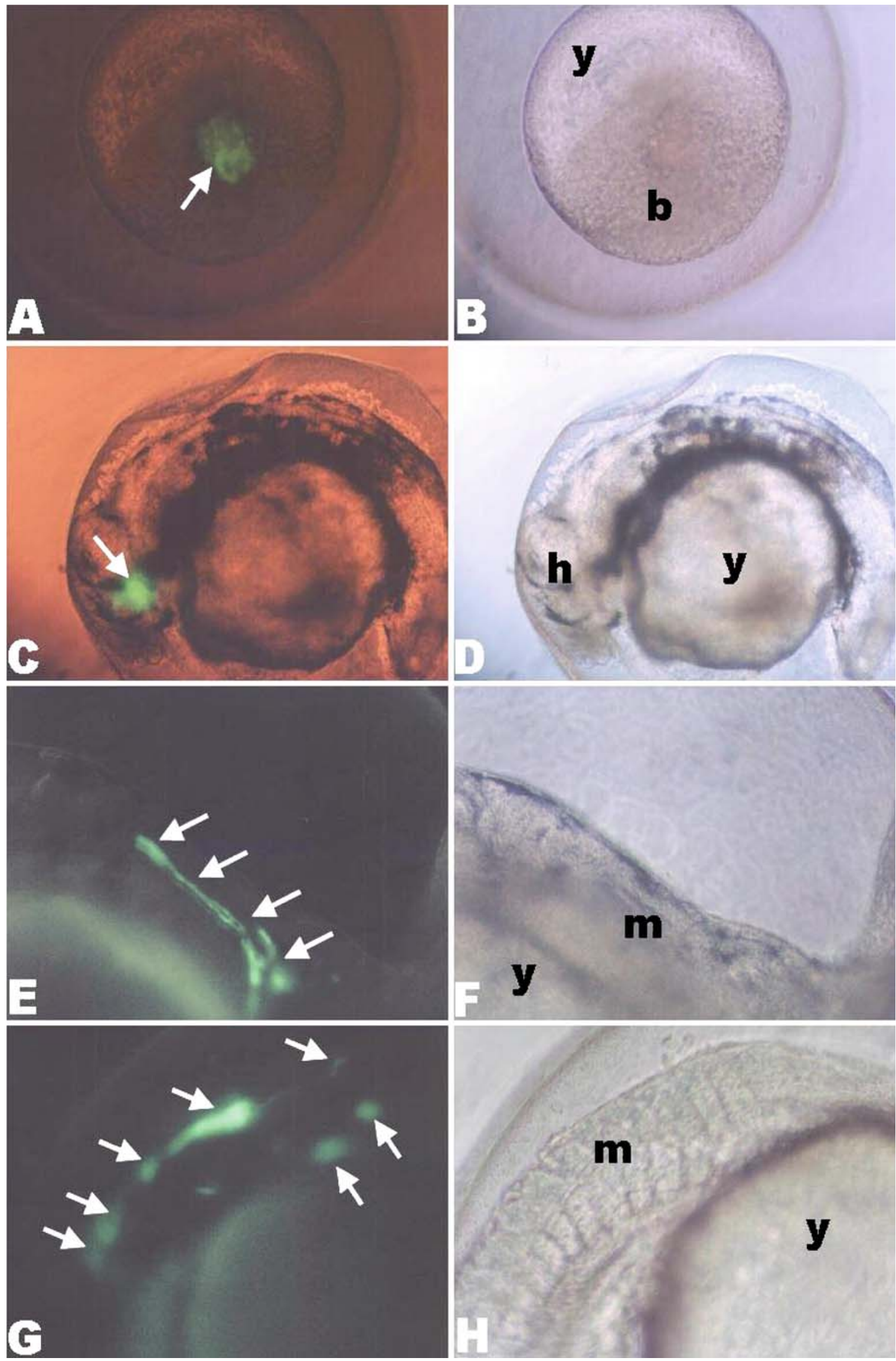

G 

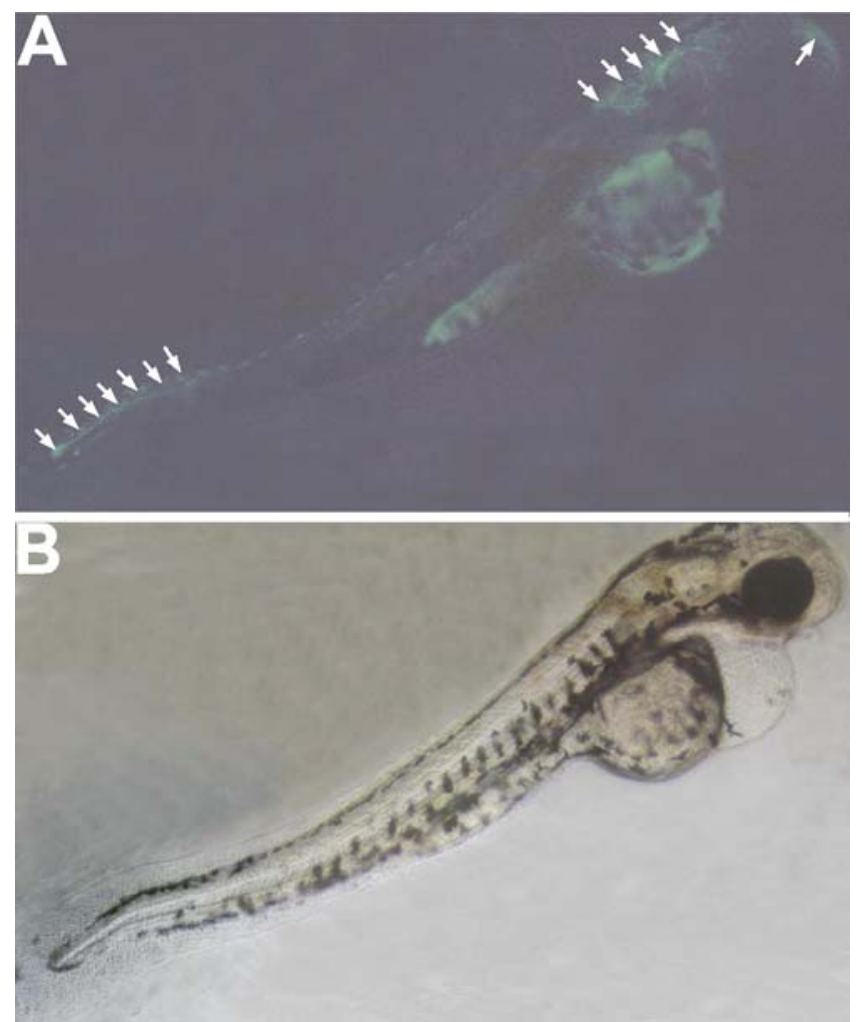

Fig. 7. Chimera fry of zebrafish obtained by transplanting GFP $^{+}$LJES1 cells into zebrafish blastula. (A, B) Fry at 3 days after hatching. GFP ${ }^{+}$LJES1 cells contributed tissues are indicated with arrows. (A) observed under a fluorescence microscope; (B) under a common microscope. Magnification: $\times 100$.

cell strain remained pluripotent and in an undifferentiated state even after gene transfection and long-term drug selection. Similar observation was made in medaka (Hong et al., 2004). These results demonstrate that gene transfection and drug selection did not influence the pluripotency and differentiation potential of LJES1 cells, which pointed to the possibility of developing gene targeting techniques through ESmediated homologous recombination in sea perch.

The transfection efficiency of foreign genes into ES cells is an important parameter for developing powerful gene targeting technique. Generally, plasmid DNA was transferred into fish ES cells by either calcium phosphate precipitation or electroporation (Hong et al., 1998b; Chen et al., 2002b). The transfection efficiency obtained via calcium phosphate precipitation was about $5 \%$ (Hong et al., 1998b). These transfection methods have the disadvantages of either low transfection efficiency or need for expensive equipment. The present study demonstrated that the Genejammer reagent resulted in high transfection efficiency $(27.3 \%)$ and is a very powerful method for transfecting LJES1 cells. This transfection method is especially suitable for the simultaneous transfection of many samples in multiwell plates and might have potential application in other fish cell transfections.

A typical feature of mouse ES cells is the ability to form EBs (Robertson, 1987). The present study demonstrated that GFP $^{+}$LJES1 cells exhibited the ability to form in vitro large EBs when the culture conditions were changed. The EB-forming ability of $\mathrm{GFP}^{+} \mathrm{LJES} 1$ cells is one important criterion for evaluating the pluripotency of LJES1 cells after GFP transfection and drug selection. Similar EBs were observed in nontransfected ES-like cell cultures in medaka (Hong et al., 1996), seabream (Bejar et al., 2002), and turbot (Holen and Hamre, 2003).

The availability of LJES1 cell strain expressing GFP lays the foundation for studying in vivo differentiation of the cells into various tissues in host embryos. GFP ${ }^{+} L J E S 1$ cells were transplanted into sea perch and zebrafish embryos. The generation of chimera embryos and fries expressing GFP from LJES1 cell transplantation confirmed the pluripotency in vivo of $\mathrm{GFP}^{+}$LJES1 cells because the cells are not only able to participate in host embryogenesis, but also differentiate into various cell types. These results are similar to other reports on ES cell transplantation into embryos of the same species (Hong et al., 1998b; Bejar et al., 2002).

Data on ES cell transplantation in fish were obtained when ES cells were transplanted into the host embryos of the same species. However, data were not available on ES cells transplantation into the recipient embryos of different species in teleost. The present study reported for the first time on the generation of chimeras via transplantation of ES cells from sea perch (Lateolabrax japonicus) belonging to Moronidae into the recipient blastulas of zebrafish (Danio rerio) belonging

Fig. 6. Chimera embryos of zebrafish obtained by transplanting GFP LJES1 cells into zebrafish blastula. $(\mathbf{A}, \mathbf{B}) \times 100$, mid-blastula embryo immediately after the injection. The cluster of GFP ${ }^{+}$LJES1 cells is indicated by arrows. (C, D) $\times 100,72$-h embryo showing a mass of GFP ${ }^{+}$LJES1 cells in head (arrow). (E, F) $\times 200$, 48-h embryo showing musclelike GFP ${ }^{+}$LJES1 cells in the trunk (arrows). (G, H) $\times 200$, 24-h embryo showing different compartments in the trunk (arrows), including neuron-like cells. (A, C, E, G) observed via fluorescence microscopy; (B, D, F, H) observed via common microscopy. 
to Cyprinidae. These results demonstrated that heterogenous ES cells (from sea perch) are able to participate in embryogenesis of zebrafish, and differentiate into various cell types in host embryos and fries, suggesting the possibility of transplanting heterogenous ES cells such as some endangered fish into the embryos of another related fish species to protect the endangered fish from extinction.

In summary, a LJES1 cell strain expressing the GFP gene $\left(\mathrm{GFP}^{+}\right.$LJES1) was developed. GFP ${ }^{+}$LJES1 in vitro can form EBs and differentiate into various cell types including muscle-like and neuron-like cells. Further, GFP ${ }^{+}$LJES1 is competent for chimera formation. Zebrafish-sea perch chimeras were generated for the first time by transplanting GFP ${ }^{+}$LJES1 cells into zebrafish blastulas. The retention of the developmental pluripotency in the sea perch $\mathrm{GFP}^{+}$LJES1 cell strain after gene transfection and drug selection lays the foundation for developing gene targeting techniques in this cultured marine fish.

\section{Acknowledgments}

This work was supported by grants from National Nature Science Foundation of China (30170740) and from State 863 High-Technology R\&D Project of China (2004AA626110).

\section{References}

Bejar J, Hong Y, Alvarez MC (2002) An ES-like cell line from the marine fish sparus aurata: characterization and chimaera production. Trans Res 11, 279-289

Chen S, Hong Y, Scherer S, Schartl M (2001) Lack of ultraviolet-light inducibility of the medakafish (Oryzias latipes) tumor suppressor gene p53. Gene 264, 197-203

Chen S, Hong Y, Schartl M (2002a) Development of a positive-negative selection procedure for gene targeting in fish cell. Aquaculture 214, 67-79

Chen SL, Hong Y, Schartl M (2002b) Structural analysis and construction of homologous recombination vectors of p53 gene from medakafish (Oryzias latipes). Acta Zool Sinica 48, 519-526

Chen SL, Sha ZX, Ye HQ (2003a) Establishment of a pluripotent embryonic cell line from sea perch (Lateolabrax japonicus) embryo. Aquaculture 218, 141-151

Chen SL, Ye HQ, Sha ZX (2003b) Derivation of a pluripotent embryonic cell line from red sea bream blastulas. J Fish Biol 63, 795-805

Collodi P, Kamei Y, Sharps A, Weber D, Barnes D (1992) Fish embryo cell cultures for derivation of stem cells and transgenic chimeras. Mol Mar Biol Biotech 1, 257-265

Evans MG, Kaufman MH (1981) Establishment in culture of pluripotential cells from mouse embryos. Nature 292, 154-156

Fan L, Crodian J, Collodi P (2004a) Culture of embryonic stem cell lines from zebrafish. Methods Cell Biol 76, 149-158

Fan L, Crodian J, Liu X, Alestrom A, Alestrom P, Collodi P (2004b) Zebrafish ES cells remain pluripotent and germ-line competent for multiple passages in culture. Zebrafish 1, 21-26

Fan L, Moon J, Crodian J, Collodi P (2006) Homologous recombination in zebrafish ES cells. Transgenic Res 15(1) 21-30

Holen E, Hamre K (2003) Towards obtaining long term embryonic stem cell like cultures from a marine flatfish, Scophthalmus maximus. Fish Physiol Biochem 29, 245-252

Hong Y, Winkler C, Schartl M (1996) Pluripotency and differentiation of embryonic stem cell lines from the medakafish (Oryzias latipes). Mech Dev 60, 33-44

Hong Y, Chen S, Winkler C, Schartl M (1998a) "Medakafish embryonic stem cells as a model for genetic improvement of aquaculture livestocks." In: New Developments in Marine Biotechnology, LeGal Y, Halvorson HO, eds. (New York: Plenum Press) pp 28-134

Hong Y, Winkler C, Schartl M (1998b) Production of medakafish chimeras from a stable embryonic stem cell line. Proc Natl Acad Sci USA 95, 3679-3684

Hong Y, Chen S, Gui J, Schartl M (2004) Retention of the developmental pluripotency in medaka embryonic stem cells after gene transfer and long-term drug selection towards for gene targeting in fish. Trans Res 13, 41-50

Lee KY, Huang H, Ju B, Yang Z, Lin S (2002) Cloned zebrafish by nuclear transfer from long-term-cultured cells. Nat Biotechnol 20(8), 795-799

Ma C, Fan L, Ganassin R, Bols N, Collodi P (2001) Production of zebrafish germ-line chimeras from embryo cell cultures. Proc Natl Acad Sci USA 98, 2461-2466

Martin GR (1981) Isolation of a pluripotent cell line from mouse embryo cultures in medium conditioned by teratocarcinoma stem cells. Proc Natl Acad Sci USA 78, 7634-7638

Melamed P, Gong Z, Fletcher G, Hew CL (2002) The potential impact of modern biotechnology on fish aquaculture. Aquaculture 204, 255-269

Mummery CL, Feyen A, Freund E, Shen S (1990) Characteristics of embryonic stem cell differentiation: a comparison with two embryonal carcinoma cell lines. Cell Differ Dev 30, 195-206

Mummery CL, Vanden Eijnden-van Raaij AJ (1993) Type beta transforming growth factors and activins in differentiating embryonal carcinoma cells, embryonic stem cells and early embryonic development. Int J Dev Biol 37, 169-182

Robertson EJ (1987) "Embryo-derived stem cell lines." In: Teratocarcinomas and Embryonic Stem Cells: A Practical Approach, Robertson EJ, ed. (Oxford: IRL Press) pp 71-112

Rocha A, Ruiz S, Estepa A, Coll JM (2004) Application of inducible and targeted gene strategies to pro- 
duce transgenic fish: a review. Mar Biotechnol 6(2), $118-127$

Sun L, Bradford CS, Ghosh C, Collodi P, Barnes DW (1995) ES-like cell cultures derived from early zebrafish embryos. Mol Mar Biol Biotechnol 4, 193-199

Thomas KR, Capecchi MR (1987) Site-directed mutagenesis by gene targeting in mouse embryo-derived stem cells. Cell 51, 503-512

Thompson S, Clarke AR, Pow AM, Hooper M, Melton DW (1989) Germ line transmission and expression of a corrected HPRT gene produced by gene targeting in embryonic stem cells. Cell 56, 313-321

Thomson JA, Itskovitz-Eldor J, Sharpiro SS, Waknitz MA, Swiergiel JJ, Marshall VS, Jones JM (1998) Embryonic stem cell lines derived from human blastocysts. Science 282, 1145-1147

Wadman SA, Clark KJ, Hackett PB (2005) Fishing for answers with transposons. Mar Biotechnol 7(3), 135-141

Wakamatsu Y, Ozato K, Sasado T (1994) Establishment of a pluripotent cell line derived from a medaka (Oryzias latipes) blastula embryo. Mol Mar Biol Biotechnol 3, 185-191

Wakamatsu Y, Ju B, Pristyaznhyuk I, Niwa K, Ladygina T, Kinoshita M, Araki K, Ozato K (2001) Fertile and diploid nuclear transplants derived from embryonic cells of a small laboratory fish, medaka (Oryzias latipes). Proc Natl Acad Sci USA 98, 1071-1076 